\title{
Regional White Matter Scaling in the Human Brain
}

\author{
${ }^{(1)}$ Allysa Warling, ${ }^{1 *}$ Cassidy L. McDermott, ${ }^{1,2 *}$ Siyuan Liu, ${ }^{1}$ Jakob Seidlitz, ${ }^{1}$ Amanda L. Rodrigue, ${ }^{3}$ Ajay Nadig, ${ }^{1}$ \\ Ruben C. Gur, ${ }^{4,5}$ Raquel E. Gur, ${ }^{4,5}$ David Roalf, ${ }^{4}{ }^{\circ}$ Tyler M. Moore, ${ }^{4,5}$ David Glahn, ${ }^{3}$ Theodore D. Satterthwaite, ${ }^{4}$ \\ ${ }^{\circ}$ Edward T. Bullmore, ${ }^{6}$ and Armin Raznahan ${ }^{1}$ \\ ${ }^{1}$ Section on Developmental Neurogenomics, Human Genetics Branch, National Institute of Mental Health, Bethesda, Maryland 20892, ${ }^{2}$ Department \\ of Psychology, School of Arts and Sciences, University of Pennsylvania, Philadelphia, Pennsylvania 19104, ${ }^{3}$ Tommy Fuss Center for \\ Neuropsychiatric Disease Research, Department of Psychiatry, Boston Children's Hospital, Harvard Medical School, Boston, Massachusetts 02115, \\ ${ }^{4}$ Department of Psychiatry, University of Pennsylvania Perelman School of Medicine, Philadelphia, Pennsylvania 19104, ${ }^{5}$ Lifespan Brain Institute of \\ the Children's Hospital of Philadelphia and Penn Medicine, Philadelphia, Pennsylvania 19104, and ${ }^{6}$ Department of Psychiatry, University of \\ Cambridge, Cambridge CB2 0SZ, United Kingdom
}

Anatomical organization of the primate cortex varies as a function of total brain size, where possession of a larger brain is accompanied by disproportionate expansion of associative cortices alongside a relative contraction of sensorimotor systems. However, equivalent scaling maps are not yet available for regional white matter anatomy. Here, we use three large-scale neuroimaging datasets to examine how regional white matter volume (WMV) scales with interindividual variation in brain volume among typically developing humans (combined $N=2391$ : 1247 females, 1144 males). We show that WMV scaling is regionally heterogeneous: larger brains have relatively greater WMV in anterior and posterior regions of cortical white matter, as well as the genu and splenium of the corpus callosum, but relatively less WMV in most subcortical regions. Furthermore, regions of positive WMV scaling tend to connect previously-defined regions of positive gray matter scaling in the cortex, revealing a coordinated coupling of regional gray and white matter organization with naturally occurring variations in human brain size. However, we also show that two commonly studied measures of white matter microstructure, fractional anisotropy (FA) and magnetization transfer (MT), scale negatively with brain size, and do so in a manner that is spatially unlike WMV scaling. Collectively, these findings provide a more complete view of anatomic scaling in the human brain, and offer new contexts for the interpretation of regional white matter variation in health and disease.

Key words: neuroimaging; scaling; white matter

\section{Significance Statement}

Recent work has shown that, in humans, regional cortical and subcortical anatomy show systematic changes as a function of brain size variation. Here, we show that regional white matter structures also show brain-size related changes in humans. Specifically, white matter regions connecting higher-order cortical systems are relatively expanded in larger human brains, while subcortical and cerebellar white matter tracts responsible for unimodal sensory or motor functions are relatively contracted. This regional scaling of white matter volume (WMV) is coordinated with regional scaling of cortical anatomy, but is distinct from scaling of white matter microstructure. These findings provide a more complete view of anatomic scaling of the human brain, with relevance for evolutionary, basic, and clinical neuroscience.

Received June 9, 2021; accepted June 12, 2021.

Author contributions: A.W., C.L.M., D.G., T.D.S., E.T.B., and A.R. designed research; A.W., C.L.M., A.L.R., D.R., T.M.M., and A.R. performed research; A.W., S.L., and A.N. analyzed data; A.W. wrote the paper; C.L.M., S.L., J.S., A.N., R.C.G., R.E.G., D.R., T.M.M., D.G., T.D.S., E.T.B., and A.R. edited the paper.

C.L.M. was supported by a National Science Foundation Graduate Research Fellowship. This work was also supported by National Institutes of Health (NIH)/National Institute of Mental Health Grants R01 MH112847 and R01 MH120482 and the National Institute of Mental Health Intramural Research Program (NIH Annual Report Number ZIAMH002949). We thank Dr. Julien Dubois for his assistance with generating g factor scores.

${ }^{*}$ A.W. and C.L.M. contributed equally to this work.

E.T.B. is a National Institute of Health Research Senior Investigator and serves as a member of the Scientific Advisory Board for Sosei Heptares and as a consultant for GlaxoSmithKline. All other authors declare no competing financial interests.

Correspondence should be addressed to Armin Raznahan at raznahana@mail.nih.gov.

https://doi.org/10.1523/JNEUROSCI.1193-21.2021

Copyright $\odot 2021$ the authors

\section{Introduction}

Human brain size varies widely among individuals of the same age (Giedd et al., 2015), and this variation is coupled with changes in the patterning of regional cortical surface anatomy. Specifically, recent work has established that larger human brains show greater areal expansion in frontoparietal association cortices (i.e., these areas exhibit hyperlinear relationships, or "positive scaling" relationships, with total brain size) at the cost of relative areal contraction in limbic and sensorimotor cortices (which have hypolinear or "negative scaling" relationships with total brain size; Reardon et al., 2018). These relationships are examples of intraspecific "static allometry" (Cheverud, 1982). Spatially similar cortical scaling has also been demonstrated as a function 
Table 1. Sample characteristics and image acquisition resolution

\begin{tabular}{llll}
\hline & Dataset & & \\
\cline { 2 - 4 } Characteristic & HCP & NSPN & PNC \\
\hline Number of individuals & 1006 & 292 & 1093 \\
Sex & $503 \mathrm{~F} / 503 \mathrm{M}$ & $147 \mathrm{~F} / 145 \mathrm{M}$ & $597 \mathrm{~F} / 496 \mathrm{M}$ \\
Age (years) & & & $14.99(3.54)$ \\
Mean (SD) & $28.24(3.54)$ & $19.11(2.87)$ & $8-23$ \\
Range & $22-35$ & $14-24$ & $0.90 \times 0.90 \times 0.90 \mathrm{~mm}$ \\
Image acquisition resolution & & $1.00 \times 1.00 \times 1.00 \mathrm{~mm}$ & $1.90 \times 1.90 \times 2.00 \mathrm{~mm}$ \\
T1 & $0.70 \times 0.70 \times 0.70 \mathrm{~mm}$ & $2.00 \times 2.00 \times 2.00 \mathrm{~mm}$ & \\
FA & $1.25 \times 1.25 \times 1.25 \mathrm{~mm}$ & $2.00 \times 2.00 \times 2.00 \mathrm{~mm}$ & \\
MT & & & \\
\hline
\end{tabular}

There was up to a 2.9-fold difference in image acquisition resolution for T1 scans. HCP = Human Connectome Project; NSPN = Neuroscience in Psychiatry Network; PNC = Philadelphia Neurodevelopmental Cohort; FA = fractional anisotropy; $\mathrm{MT}=$ magnetization transfer.

of brain size variation across primates (evolutionary allometry), and within humans over development (ontogenetic allometry; Hill et al., 2010), hinting at a conserved relationship between brain size and regional cortical patterning in primates. Notably, positively-scaling cortical regions in humans tend to be specialized for integrative functions and long-range corticocortical connectivity (Margulies et al., 2016; Reardon et al., 2018).

Here, we address a currently unexamined hypothesis which is raised by these gray matter results: whether regionally-heterogeneous anatomic scaling is also seen in white matter. Specifically, we hypothesize that, just as some cortical regions are relatively expanded and others contracted in larger human brains, there will also be regions of positive and negative white matter scaling. If white matter scaling coheres with previously-described gray matter scaling (Reardon et al., 2018), we would expect positive scaling in tracts underlying association cortices, and negative scaling in tracts underlying sensorimotor cortices. However, with the notable exception of the corpus callosum (Jancke, 1997; Karolis et al., 2019), we have little prior data about scaling of major interhemispheric or intrahemispheric tracts to inform these predictions. It is well established that global white matter volume (WMV) scales non-linearly (positively) with brain size variation within humans (Lüders et al., 2002; de Jong et al., 2017), but it remains unknown whether WMV scaling varies between regions. Thus, we cannot yet comprehensively test the current dominant theory that increased brain size should be accompanied by relative increases in intrahemispheric connectivity, alongside relative decreases in interhemispheric connectivity (Jancke, 1997; Karolis et al., 2019). A comprehensive map of regional WMV scaling in humans would address this theory, while also providing new frameworks for future research on white matter changes in health (Walhovd et al., 2005; Lenroot et al., 2007) and disease (Blumenthal et al., 2013; Villalón-Reina et al., 2020) and complementing our knowledge of gray matter scaling (Reardon et al., 2018).

This study uses an allometric framework to systematically characterize regional WMV scaling as a function of normative brain size [or total tissue volume (TTV)] variation. We first leverage three large-scale human neuroimaging datasets to produce and replicate WMV scaling norms and establish sets of white matter regions that scale differently with TTV variation. These datasets involved different scanners, image resolutions, and participant cohorts (Table 1). We next characterize the heritability and cognitive associations of WMV within these regions. Further, we demonstrate that WMV scaling is uncoupled from that of two biologically-salient (Mädler et al., 2008; Hagiwara et al., 2018) markers of white matter microstructure, fractional anisotropy (FA) and magnetization transfer (MT), both of which are known to increase (in absolute terms) in larger brains (Takao et al., 2011), but about which we have little preexisting knowledge of relative scaling relationships. Finally, we harness an independent tractography atlas (Zhang and Arfanakis, 2018) to integrate our WMV scaling findings with preexisting gray matter scaling maps (Reardon et al., 2018) and show that TTV variation is associated with an orchestrated expansion of WMV in associative cortical tracts alongside a relative contraction of WMV within sensorimotor systems. These findings provide an integrated view of scaling across both major tissue classes of the brain, revealing that larger brains not only show a preferential expansion of associative cortices, but also of the white matter tracts that interconnect them.

\section{Materials and Methods}

\section{Participant recruitment and MRI data acquisition}

Human connectome project (HCP)

Our primary cohort of 1006 typically-developing young adults $\left(\mathrm{M}_{\text {age }}=\right.$ $28.2 \pm 3.5$; range $=22-35$; see Table 1 ) was drawn from the HCP 1200 release (Van Essen et al., 2013). Participants were recruited from a group of adult twins and their non-twin siblings. Families were screened with phone interviews, and exclusionary criteria included severe neuropsychiatric or neurodevelopmental disorders, diabetes, hypertension, and early gestation age. Informed consent was obtained from all participants, and the procedures were approved by the Washington University Institutional Review Board. Data are from the HCP S1200 release (https://humanconnectome. $\operatorname{org} /)$.

All participants had T1-weighted structural MRI scans; 966 of these participants also had diffusion-weighted imaging (DWI) scans. Scans were acquired over a 2-d period at Washington University using a custom Siemens 3T "Connectome Skyra" scanner with a 32-channel radio frequency (RF) headcoil (Siemens Healthcare). For T1-weighted structural scans, two separate averages of a 3D magnetization prepared, rapid-acquisition gradient-echo (3D MRPAGE) sequence with $0.7-\mathrm{mm}$ isotropic resolution were collected $(\mathrm{FOV}=224 \mathrm{~mm}$, matrix $=320$, $\mathrm{TR}=2400 \mathrm{~ms}, \mathrm{TE}=2 / 14 \mathrm{~ms}, \mathrm{TI}=1000 \mathrm{~ms}, \mathrm{FA}=8^{\circ}$; Van Essen et al., 2013). For DWI scans, a high-angular resolution diffusion-weighted image (HARDI) was acquired using three shells of $b=1000,2000$, and $3000 \mathrm{~mm} / \mathrm{s}^{2}$, over many directions (270 q-points over the $3 \mathrm{~b}$-shells; as well as 18 B0 images; Glasser et al., 2013; Uğurbil et al., 2013; Van Essen et al., 2013). More comprehensive explanations of scanning procedures are available elsewhere (Glasser et al., 2013).

\section{Philadelphia neurodevelopmental cohort (PNC)}

We included 1093 children and young adults $\left(\mathrm{M}_{\mathrm{age}}=15.0 \pm 3.5\right.$; range $=8-23$; see Table 1) from a 50,000 person cohort at the center for Applied Genomics at the Children's Hospital of Philadelphia (Satterthwaite et al., 2014) to test for replicability of our findings in the primary HCP dataset (Table 2). Participants were recruited by mail and screened over the 
Table 2. Normative scaling relationships between global volumes, mean FA, mean MT, and brain size

\begin{tabular}{|c|c|c|c|c|}
\hline & & \multicolumn{3}{|l|}{ Dataset } \\
\hline & & $\mathrm{HCP}$ & NSPN & PNC \\
\hline Global WMV & $\beta_{1}$-scaling coefficient (SE) & $1.10(0.018)^{* * *}$ & $1.10(0.032)^{* *}$ & $1.06(0.018)^{* *}$ \\
\hline Global GMV & $\begin{array}{l}\beta_{1 \text {-scaling coefficient (SE) }} \\
p\end{array}$ & $\begin{array}{l}0.94(0.011)^{* * *} \\
6.3 \times 10^{-8}\end{array}$ & $\begin{array}{l}0.95(0.016)^{* *} \\
0.005\end{array}$ & $\begin{array}{l}0.96(0.010)^{* * *} \\
3.6 \times 10^{-4}\end{array}$ \\
\hline Mean FA & $\beta_{1}$-scaling coefficient (SE) & $\begin{array}{c}0.10(0.014)^{* * *} \\
1 \times 10^{-5}\end{array}$ & $\begin{array}{l}0.11(0.021)^{* * * *} \\
2 \times 10^{-16}\end{array}$ & $\begin{array}{l}0.07(0.014)^{* * * *} \\
1.8 \times 10^{-8}\end{array}$ \\
\hline
\end{tabular}

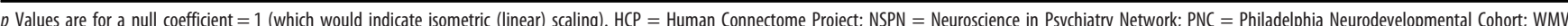
$=$ white matter volume; GMV = gray matter volume; $\mathrm{FA}=$ fractional anisotropy; $\mathrm{MT}=$ magnetization transfer. ${ }^{*} p<0.05,{ }^{* *} p<0.01,{ }^{* * *} p<0.001$.

phone. Exclusionary criteria included a history of psychiatric medication or hospitalization, medical issues that could impact brain function or the ability to finish neuroimaging tasks, and implanted metal. Written informed consent/assent was given by all participants, including parental consent for pediatric patients. The study was approved by the Institutional Review Boards of both the University of Pennsylvania and the Children's Hospital of Philadelphia. PNC genotypes and phenotypes are available (dbGaP accession: phs000607.v2. p2).

All participants had T1-weighted structural MRI scans and DWI scans. Structural scans were acquired using a 3T Siemens TIM Trio whole-body scanner (Siemens Healthcare) at the University of Pennsylvania. Images were obtained with an MPRAGE sequence (FOV $=180 \mathrm{~mm}$, acquisition matrix $=192,256160, \mathrm{ET}=3.5 \mathrm{~ms}, \mathrm{RT}=1810 \mathrm{~ms}, \mathrm{FA}=9^{\circ}$ ). DWI scans were captured with a twice-refocused spin-echo (TRSE) single-shot EPI sequence, consisting of 64 diffusion-weighted directions with $\mathrm{b}=1000 \mathrm{~s} /$ $\mathrm{mm}^{2}$, and seven interspersed scans where $\mathrm{b}=0 \mathrm{~s} / \mathrm{mm}^{2}$ (Roalf et al., 2016). Complete details about scanning acquisition are available elsewhere (Satterthwaite et al., 2014; Roalf et al., 2016).

\section{Neuroscience in psychiatry network (NSPN)}

We included 292 typically developing adolescents and young adults $\left(\mathrm{M}_{\text {age }}=19.1 \pm 2.9\right.$; range $=14-24$; see Table 1$)$ from the NPSN cohort for further replication analyses of WMV scaling findings in the primary HCP dataset, and for integrative analysis of WMV scaling alongside scaling of FA and MT (Table 2). Participants were recruited for an NSPN study on typical adolescent development (Kiddle et al., 2018; Seidlitz et al., 2018). Exclusionary criteria included current treatment for a psychiatric disorder or drug or alcohol dependence, current or past history of neurologic disorders or trauma, head injury with loss of consciousness, and treatment for a learning disability. Written informed consent was obtained from all participants, and parental consent was given for those aged 14-15. The study was approved by the National Research Ethics Service, and conducted according to National Health Service research standards. Data will be made available on reasonable request to openNSPN@medschl.cam.ac.uk.

All included participants had T1-weighted structural MRI, DWI, and MT data. Structural scans, including MT data, were acquired with the multi-parametric mapping sequence (Kiddle et al., 2018; Seidlitz et al., 2018) on three identical 3T scanners (Magnetom TIM Trio, Siemens Healthcare; VB17 software version). Each scanner had a standard 32channel (RF) headcoil. R1 and MT were quantified in MATLAB (The MathWorks Inc.) using SPM8 (www.fil.ion.ucl.ac.uk/spm). DWI data were collected in the same session: a HARDI was acquired using a single-shot echoplanar imaging (EPI) sequence, with 63 gradient directions and $b$ value $=1000 \mathrm{~mm} / \mathrm{s}^{2}$ (as well as five unweighted B0 images). More comprehensive explanations of scanning procedures are available (Weiskopf et al., 2013; Kiddle et al., 2018; Seidlitz et al., 2018).

\section{Data availability statement}

HCP data are from the HCP S1200 release and can be found on the study website (humanconnectome.org); PNC data are available for access (dbGaP accession: phs000607.v2. p2); and NSPN data are managed by NSPN but will be made available on reasonable request to openNSPN@medschl.cam.ac.uk.

\section{Image processing}

To carry out deformation-based morphometry (DBM) analyses, we applied the diffeomorphic anatomical registration through exponentiated lie algebra (DARTEL) procedure (SPM12 software package; Ashburner, 2007) separately to $\mathrm{T}_{1}$ images in the HCP, NSPN, and PNC datasets. DARTEL is a widely used nonlinear deformation algorithm that provides high registration accuracy (Klein et al., 2009). In brief, images were corrected for magnetic field inhomogeneity. Then, taking advantage of built-in tissue probability maps for gray matter, white matter, cerebrospinal fluid, bone, soft tissue, and air/background, images were segmented and normalized to Montreal Neurologic Institute (MNI) space in a unified model (Ashburner and Friston, 2005). Following this, DARTEL-imported versions of both these rigid-body aligned gray and white matter probability maps without linear scaling in individual space were used to generate flow fields for each voxel and encode deformation, as well as a series of population template images. During this step, DARTEL increases the accuracy of intersubject alignment by nonlinearly registering individual gray and white matter simultaneously to the template. As the data are iteratively aligned, the template becomes increasingly crisp. Using the final flow fields and template image, the gray and white matter probability (or density) images in individual space were spatially normalized and resliced to isotropic voxel size in MNI space, modulated/Jacobian scaled (i.e., multiplied by the Jacobian determinant of the deformation field from template to individual space), and then smoothed with a 4-mm full-width at half-maximum (FWHM) Gaussian Kernel.

All normalized HCP, NSPN, and PNC images were visually inspected to ensure absence of any visible motion artifacts or inaccurate segmentation and normalization. Those smoothed, modulated, normalized WMV probability maps were used for the following scaling analyses. Using the same flow fields generated by DARTEL, FA and MT maps were normalized from individual space to MNI space and smoothed with the same 4-mm FWHM, Gaussian Kernel.

\section{Details of DWI data preprocessing \\ HCP}

EPI, eddy-current induced field inhomogeneities, and motion were estimated and corrected using FSL (Glasser et al., 2013). The impact of gradient nonlinearity on the diffusion vectors was calculated and applied to correct them at each voxel. The corrected images were transformed and registered to native structural space. Further details are published (Glasser et al., 2013; Sotiropoulos et al., 2013).

\section{PNC}

Manual and automated quality assurance procedures were applied to remove problematic DWI data (Roalf et al., 2016). DWI data were skull stripped and eddy currents and motion were estimated and corrected using FSL's eddy. Motion output generated from eddy was used to adjust gradient vectors for motion. The field map was estimated and distortion correction was applied. Finally, the diffusion tensor was modeled and 
FA was estimated at each voxel using FSL's DTIFIT. Full analytical details are published (Roalf et al., 2016).

\section{NSPN}

The DWI image analysis pipeline was performed in AFNI (Cox, 1996). For each subject, DWI image volumes were de-obliqued and co-registered to the B0 volume to account for in-scanner motion. Then, the six principal direction tensors were estimated from the DWI image volumes using the $3 \mathrm{dDWItoDT}$ command. A combinatorial scalar measure derived from these directions (eigenvalues of the tensor), FA, was used for further analysis.

\section{Experimental design and statistical analysis Statistical models}

Voxel-wise estimation of white matter structure scaling. We used log-log regression (Huxley, 1924) to determine the normative allometry of white matter structure relative to total brain size in typically developing males and females. Under this framework, the scaling relationship of metric $\mathrm{A}$ relative to metric $\mathrm{B}$ is given by $\beta 1$ in the following equation:

$$
\log 10(A)=\beta 0+\beta \log 10(B) .
$$

Using this formula, we estimated the nonlinear allometric relationship between WMV at each voxel (i.e., metric A), and TTV (i.e., metric B). Here, a coefficient of 1 indicates linear (or isometric) scaling, while coefficients significantly different from 1 indicate nonlinear scaling. Specifically, values significantly less than 1 indicate a negative scaling relationship, where A becomes proportionally smaller as B increases (i.e., the rate of increase of A does not keep up with the rate of increase of $\mathrm{B}$ ). Conversely, values significantly $>1$ indicate a positive scaling relationship, where $\mathrm{A}$ becomes proportionally larger as B increases.

Our primary dependent variables of interest were WMV at each voxel within a white matter mask. The white matter mask applied included only voxels for which the cohort average white matter density was $>0.6$ (i.e., separate masks were generated for each of the three datasets). Our independent variable used to predict WMV was TTV, which was computed as the sum of total WMV and total gray matter volume (GMV). As there are known age-based and sex-based differences in absolute measures of cortical and subcortical anatomy, we removed age-related and sex-related variation in local WMV and in TTV using nonlinear splines. In order to avoid negative values, the volumes at mean age were added to the age-regressed variables. These age-residualized volumetric variables were then logged in the allometric equation described above.

We then tested for potential interactive effects of scaling, sex, and centered age, to examine whether age and sex affected anatomic scaling relationships, for all allometric scaling models using the following model:

$$
\begin{gathered}
\log 10(A)=\beta 0+\beta 1 \log 10(B)+\beta 2(S E X)+\beta 3(A G E) \\
+\beta 4(\log 10(B) \times S E X)+\beta 5(\log 10(B) \times A G E)+\beta 6(S E X \times A G E) \\
+\beta 7(\log 10(B)) \times S E X \times A G E) .
\end{gathered}
$$

This model allowed us to rule out significant interactions between these variables [i.e., none of the interaction terms, 4, 5, 6, or 7, survived false discovery rate (FDR) correction]. Furthermore, we found no significant main effect of age (3) on any dependent variable, so we dropped age from the model. Thus, we present results from the following simplified allometric scaling model:

$$
\log 10(A)=\beta 0+\beta 1 \log 10(B)+\beta 2(\text { SEX }) .
$$

Voxel-wise scaling coefficients were visualized on the MNI-152 template brain (Van Essen et al., 2013), and sagittal, coronal, and axial slices were selected for visualization. Regions of statistically significant positive and negative scaling were defined by testing the observed value against a null hypothesis of 1 (isometric scaling) and applying a FDR correction for multiple comparisons across all voxels in the white matter mask with $q$ set to 0.05 . In the manuscript we present both (1) the unthresholded scaling maps in each dataset and (2) the thresholded, binarized scaling maps after FDR correction. In each figure, regions of positive scaling are visualized in red, and regions of negative scaling are visualized in blue. Tracts were identified by comparing these scaling maps to a stereotaxic DTI-based white matter atlas in the same (MNI) space (Mori et al., 2008).

The log-log regression methods described above were also used to estimate voxel-specific scaling of FA and MT relative to TTV in the NSPN cohort only (as that was the only cohort where both FA and MT information was available). We also conducted two additional supplementary analyses in the NSPN dataset to further qualify our main findings. First, to test for consistency of WMV, FA, and MT findings across different image resolutions, we compared scaling results from our original models in the NSPN cohort, which used images with $1.5-\mathrm{mm}^{3}$ voxel sizes for all three modalities, to scaling results using images with 1 - and $2-\mathrm{mm}^{3}$ voxel sizes. This involved repeating the voxel-wise scaling analyses above on T1-weighted structural MRI, DWI, and MT scans that had been normalized and resliced to $1-$ and $2-\mathrm{mm}^{3}$ voxel sizes in MNI space using the same deformation field determined above. Continuous and thresholded scaling coefficient maps were compared and visualized across all three voxel sizes (for continuous maps, see Warling, 2021a). Second, given that some studies of brain allometry (especially analyses of interspecies scaling; Ventura-Antunes et al., 2013) exclude the independent variable from global size estimates, we also estimated global and regional WMV scaling in the NSPN dataset with total GMV rather than with TTV.

\section{Comparison of white matter scaling maps}

We calculated Pearson correlation coefficients to quantify the consistency of scaling maps between cohorts within the same modality (e.g., correlation between HCP WMV scaling map and PNC WMV scaling map), as well as the consistency of scaling maps across the two modalities within the same cohort when appropriate (e.g., correlation between NSPN WMV scaling map and NSPN FA scaling map). For correlations between cohorts, only the voxels that overlapped in the two white matter masks were included in the analysis. For each correlation, significance was assessed using permutation testing: for each cohort of interest, the independent variable (age-residualized TTV) was randomly shuffled and new allometric scaling maps were calculated. A null distribution was generated from 1000 correlations of permuted scaling maps and was then tested against the actual correlation of the two non-permuted scaling maps.

\section{Heritability and cognitive analyses in the HCP}

To benchmark those regions of nonlinear WMV scaling against the rich and growing literature of heritability of white matter phenotypes (Schmitt et al., 2020), we calculated the heritability $\left(h^{2}\right)$ of TTV, WMV, GMV, and regions of significantly positive and negative WMV scaling with SOLAR v8.1.1 within the HCP dataset (Almasy and Blangero, 1998). This tool employs maximum likelihood variance decomposition methods to determine the relative effects of genetic and environmental influences by modeling the covariance among family members as a function of genetic proximity. Specifically, heritability is the proportion of trait variance that is explained by additive genetic factors, and is estimated by partitioning the phenotypic covariance matrix into genetic and environmental components as $h 2=\sigma G 2 /(\sigma G 2+\sigma \mathrm{E} 2)$, where $\sigma_{G}^{2}$ represents additive genetic variance and $\sigma_{E}^{2}$ represents random environmental effects. The covariance matrix, $\Omega$, is composed of the $\mathrm{n} \times \mathrm{n}$ kinship matrix (R) and an $\mathrm{n} \times \mathrm{n}$ identity matrix (I): $\Omega=2 \mathrm{R}{\sigma_{E}}^{2}+\mathrm{I} \sigma_{E}^{2}$. Tests of heritability are performed using standard likelihood ratio tests in which the ln likelihood of the null model (where the focal variance component is forced to be zero) is compared with that of the alternative model (where the focal variance component is explicitly estimated from the data). Twice the difference in these ln likelihoods yields a likelihood 


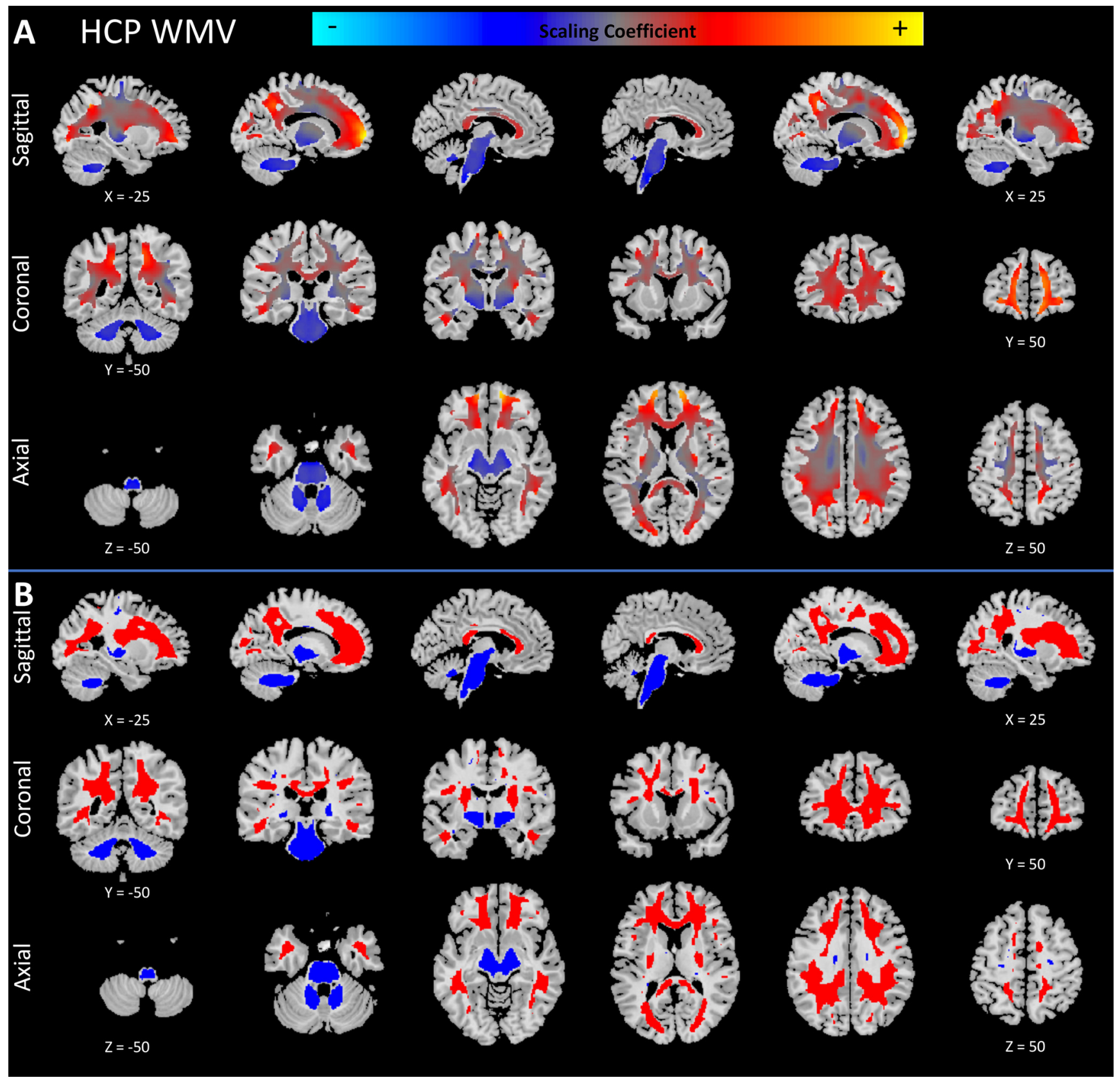

Figure 1. Nonlinear scaling of local white matter volume (WMV) with brain size in the Human Connectome Project (HCP) cohort. A, Sagittal, coronal, and axial slices displaying the unthresholded map of local WMV scaling with Total tissue volume (TTV) in the HCP cohort. Red indicates relatively greater WMV with larger TTV ("positive" scaling), and blue indicates relatively smaller WMV with larger TTV ("negative" scaling). B, Categorical scaling map showing regions of statistically significant positive and negative WMV scaling after correction for multiple comparisons across voxels.

ratio test statistic that is asymptotically distributed as a 50:50 mixture of a $\chi^{2}$ variate with one degree of freedom and a point mass at zero. We did not partition $\sigma_{E}^{2}$ into unique and shared environmental components given the small size of the observed combined environmental components (see below). Before conducting heritability analyses, an inverse-norm transformation was applied to each trait. All heritability models included the following covariates: age, age $^{2}$, sex, and their interactions (age $\times$ sex and age $e^{2} \times$ sex). Heritability of WMV scaling traits was also calculated with and without residualizing measures for TTV.

Interrelating cortical and white matter scaling

We employed the IIT Human Brain Atlas (Zhang and Arfanakis, 2018) to identify pairs of positively-scaling gray matter regions (Reardon et al., 2018) that were also connected through regions of positive white matter scaling in the HCP. The IIT atlas provides probabilistic estimates of regional interconnectivity for all major white matter tracts in a cohort that is independent of those we used to map WMV scaling. Moreover, IIT regional interconnectivity estimates are provided for those same gray matter regions for which we had previously-established indices of surface-based scaling with total brain size (Reardon et al., 2018). Thus, we were able to identify overlapping WMV and GMV scaling changes. We defined the white matter ROIs as a mask of all white matter voxels that showed significant positive WMV scaling in the HCP in our present analyses. The IIT Atlas resources include a connectivity matrix that contains the absolute number of white matter "streamlines" connecting each pair of gray matter regions in the Desikan-Killiany Atlas. Working from this matrix, we calculated the percent of those connecting streamlines that also passed through our positively-scaling white matter ROIs. We 


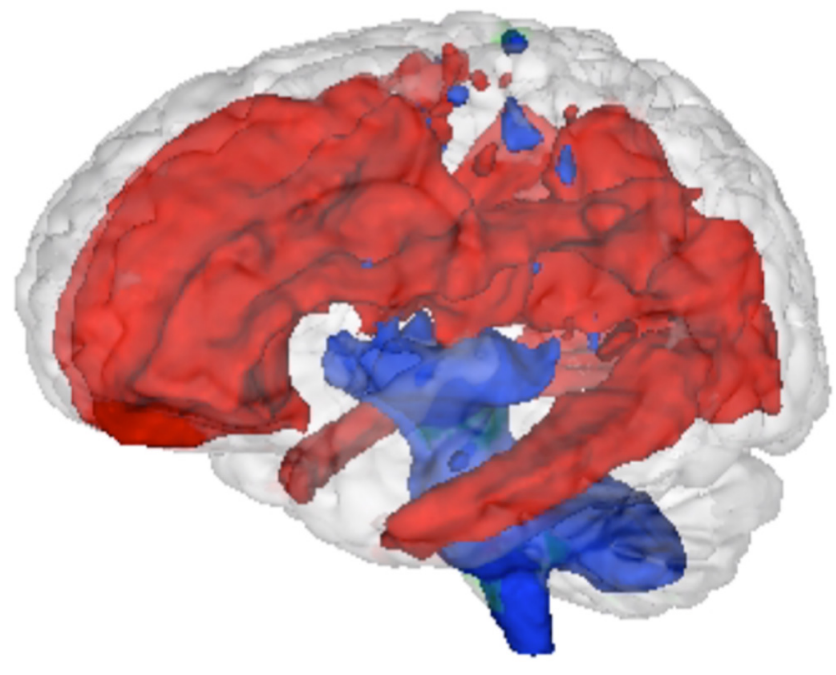

Movie 1. Visualization of voxels with significantly non-linear WMV scaling in the Human Connectome Project (HCP) cohort. Colored regions are those that show significant positive (red) or negative (blue) white matter volume (WMV) scaling ( $p>0.05$ after FDR correction) with total tissue volume (TTV) in the HCP. A 2D image of positively-scaling voxels from this visualization is included in Figure $6 B$. [View online]

compared the magnitude of these white matter percentages to the number of positively-scaling vertices in a previously-defined set of gray matter regions that themselves scaled positively with TTV (Reardon et al., 2018). We also further characterized the positively-scaling white matter connections between gray matter regions with at least $50 \%$ positivelyscaling vertices by visually mapping those connections. All analyses were completed in R 3.6.0 (R Core Team, 2013) using the oro.nifti, voxel, gtools, ggplot2, mgcv, RccpEigen, and snow packages (Whitcher et al., 2011; Wood, 2011; Bates and Eddelbuettel, 2013; Wickham, 2016; Garcia de la Garza et al., 2018; Tierney et al., 2018; Warnes et al., 2020).

\section{Results}

\section{Reproducible patterns of nonlinear regional WMV scaling in} humans

As a context for the examination of regional WMV scaling, we first verified prior reports that total WMV scales positively with global brain size variation in humans (Lüders et al., 2002; de Jong et al., 2017) across our three datasets. Application of a loglog regression approach (Huxley, 1924) to quantify the scaling relationship between global WMV and TTV (calculated as the sum of total WMV and total GMV for each individual) confirmed that total WMV showed positive scaling with TTV across all datasets analyzed (i.e., scaling coefficients for HCP, NSPN, and PNC datasets were all significantly $>1$; Table 2 ). These analyses controlled for age and sex (neither of which modified the observed scaling relationships), and indicated that individuals with larger brains had proportionally greater WMV. As follows from the WMV-TTV scaling relationship, total GMV showed negative scaling with TTV (i.e., scaling coefficients significantly $<1$; Table 2). Although total WMV and total GMV both showed statistically-significant deviations from linear scaling with TTV, the effect size of these deviations was small (Table 2).

Next, to test our core hypothesis that WMV scaling relationships with TTV differ across brain regions, we applied the same log-log regression model to relate WMV variation at each voxel within the white matter mask to variation in TTV. These voxelwise analyses revealed marked regional variability of white matter scaling in the primary HCP dataset, with scaling coefficients ranging from 0.54 (i.e., extreme negative scaling coefficient furthest below the linear scaling coefficient value of 1 , localizing to the brainstem) to 2.09 (extreme positive scaling coefficient furthest above 1, localizing to the frontal pole; Fig. $1 A$ ). After FDR correction for multiple comparisons across voxels, we observed regions of statistically-significant negative WMV scaling in brainstem and cerebellar tracts, as well as a medial subcortical region that contains the posterior limb of the internal capsule (Fig. 1B; Movie 1). Regions of statistically-significant positive WMV scaling were more widespread throughout the cerebrum, and included sections of the prefrontal white matter, anterior corona radiata, corpus callosum (genu and splenium), cingulum, and the superior longitudinal fasciculus (Fig. 1B; Movie 1). We verified the reproducibility of this voxel-level WMV scaling map by regenerating it in each of 1000 randomly generated splithalves of the HCP dataset, and establishing that the mean crossvoxel correlation in WMV scaling coefficients between maps from each half was high $\left(r_{\text {mean }}=0.88\right)$.

The observed map of regional WMV scaling from analysis of the primary HCP dataset could also be robustly replicated by independent application of our analytic workflow to the NSPN and PNC datasets (Fig. 2), which, notably, cover different age ranges and scanning platforms. Specifically, voxel-wise WMV scaling coefficients were highly correlated between each pair of datasets $\left(r_{H C P-N S P N}=0.81, r_{H C P-P N C}=0.90, r_{N S P N-P N C}=0.76\right.$; all ps $<2.2 \times 10^{-16}$; Fig. 3). Moreover, a conjunction map of statistically-significant nonlinear WMV scaling from the HCP, NSPN, and PNC cohorts revealed a pattern of overwhelmingly convergent WMV scaling among the three datasets (Fig. 3), reinforcing the bilaterally symmetric regions of significant positive and negative WMV scaling found in analysis of the primary HCP dataset.

Having identified clusters of consistently positive and negative WMV scaling across all three datasets, we next used information on relatedness and cognitive functioning in the HCP dataset to investigate the heritability and potential cognitive correlates of WMV variation within these clusters (Materials and Methods) as defined in the HCP dataset (Fig. 1B). Estimated heritability was high for TTV, GMV, and WMV $\left(h^{2}=0.96, h^{2}=\right.$ 0.95 , and $h^{2}=0.94$, respectively), as well as WMV within both the positive and negative scaling clusters defined in the HCP dataset $\left(h^{2}=0.95\right.$ and $h^{2}=0.96$, respectively). The heritability values for TTV, as well as global GMV and WMV, are generally in line with previously published values (Peper et al., 2007) and were comparable to heritability estimates for other variables, such as height, in this dataset (see Warling, 2021b). Heritability remained high for TTV-residualized WMV variation within positive and negative scaling clusters $\left(h^{2}=0.96\right.$ and $h^{2}=0.92$, respectively). A summary score of general intelligence derived from the HCP dataset (Dubois et al., 2018; henceforth, $g$ ) showed a moderately strong positive correlation with TTV, GMV, and WMV $\left(r=0.31, p=2.2 \times 10^{-16} ; r=0.34, p=2.2 \times 10^{-16} ; r=0.22\right.$, $p=2.0 \times 10^{-11}$, respectively), as well as WMV volume within both the positively-scaling and negatively-scaling clusters defined in the HCP dataset $\left(r=0.22, p=2.9 \times 10^{-11} ; r=0.21, p=1.2 \times 10^{-10}\right.$, respectively). However, variation in WMV after residualization for TTV variation revealed weaker, negative correlations with $\mathrm{g}$ (total WMV: $r=-0.18, p=1.1 \times 10^{-07} /$ positively-scaling WMV regions: $r=-0.18, p=6.9 \times 10^{-08} /$ negatively-scaling WMV regions: $r=$ $-0.07, p=0.03$ ). Independent analysis with the PNC dataset using a factor analytically- derived index of cognition (Gur et al., 2021) failed to reveal a statistically significant correlation between general cognitive ability and WMV in either positively-scaling or negatively-scaling WMV clusters. 


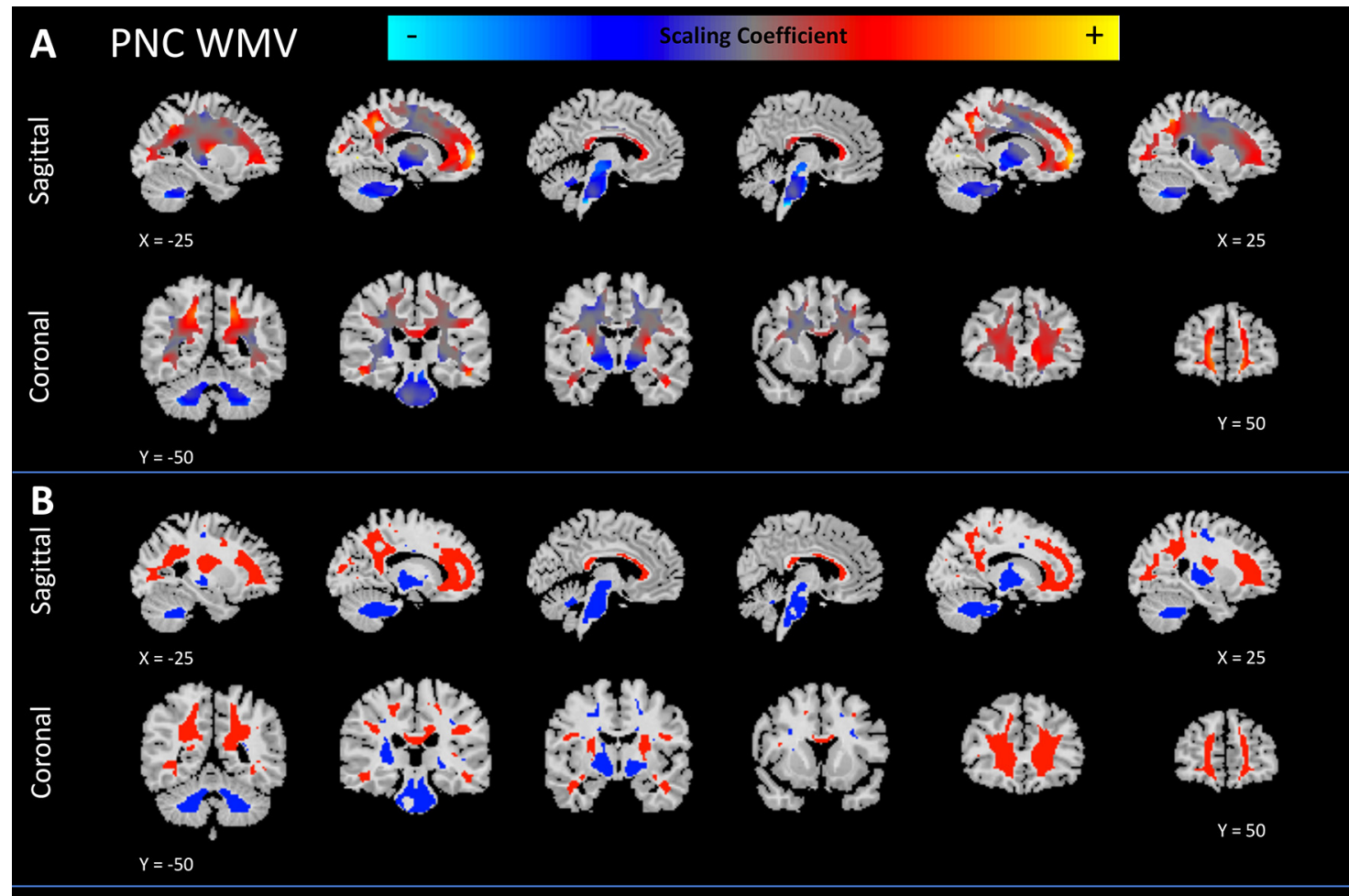

C NSPN WMV

$\frac{\pi}{5}$
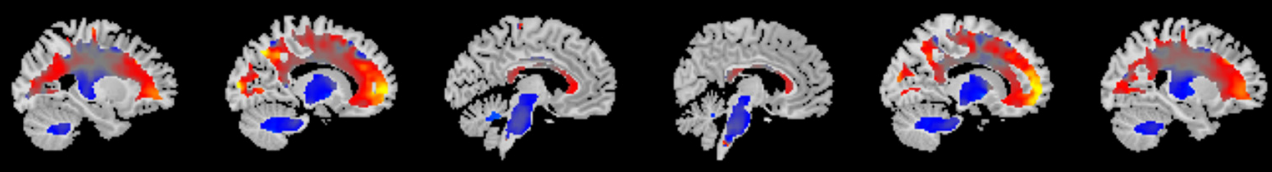

$X=-25$
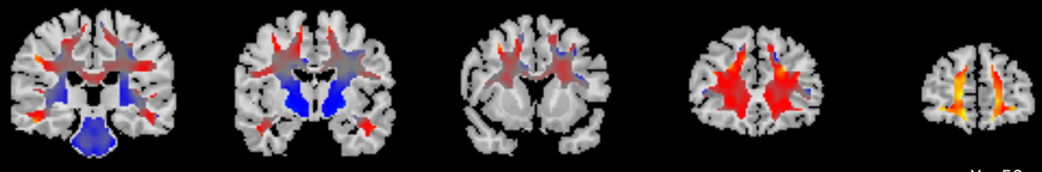

$\mathrm{Y}=50$

$\mathrm{Y}=-50$

$\frac{D}{\frac{D}{\mathbb{7}}}$
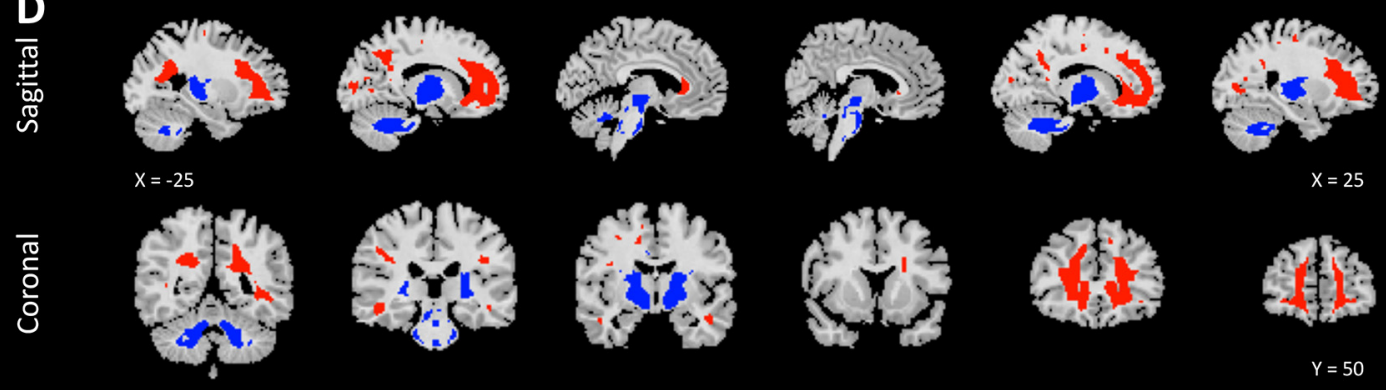

$Y=-50$

Figure 2. Nonlinear scaling of local WMV with brain size in the Neuroscience in Psychiatry Network (NSPN) and Philadelphia Neurodevelopmental Cohort (PNC) cohorts. A, Unthresholded map showing local white matter volume (WMV) scaling with total tissue volume (TTV) in the PNC cohort. B, Categorical scaling map showing regions of statistically significant positive and negative WMV scaling in the PNC cohort, after correction for multiple comparisons across voxels. C, Unthresholded map showing local WMV scaling with TTV in the NSPN cohort. D, Categorical scaling map showing regions of statistically significant positive and negative WMV scaling in the NSPN cohort, after correction for multiple comparisons across voxels.

Regional scaling of WMV in humans is uncoupled from that of white matter microstructure

The results above demonstrate that regional distribution of WMV is altered as a function of brain size variation in humans, but do not establish whether these volumetric effects are accompanied by microstructural changes. Therefore, we next applied the same log-log regression models used in WMV analyses to clarify scaling relationships between TTV and two commonlystudied neuroimaging markers of white matter microstructure: FA and MT. FA may reflect diverse microstructural properties, including axonal integrity, fiber density, tract crossing, and/or myelination (Beaulieu, 2002), and MT is considered a more 


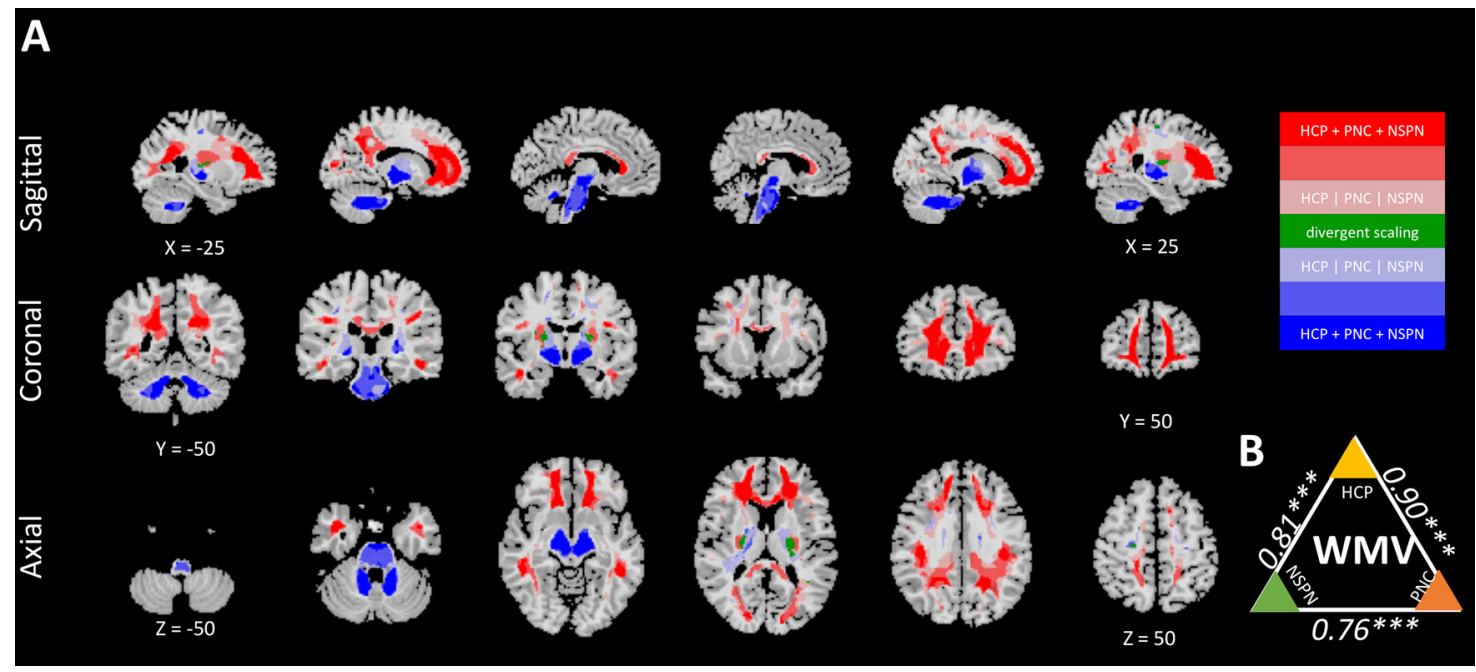

Figure 3. Conjunction of local white matter volume (WMV) scaling patterns across datasets. $\boldsymbol{A}$, Conjunction of Human Connectome Project (HCP), Neuroscience in Psychiatry Network (NSPN), and Philadelphia Neurodevelopmental Cohort (PNC) white matter scaling maps. A small, bilateral region of divergent scaling was identified in the internal capsule (shown in green), bordered by a region of consistent negative scaling medially, and a region of consistent positive scaling laterally. $\boldsymbol{B}$, Cross-voxel correlations between WMV scaling coefficients in the HCP (yellow), NSPN (green), and PNC (orange) cohorts (all ps $<2.2 \times 10^{-16}$ ).

robust marker of myelin (Lema et al., 2017). Modality availability across datasets was such that FA analyses could be completed in all three cohorts, and MT analyses in the NSPN cohort only.

In contrast to the consistently positive scaling relationships between total WMV and TTV (Table 2), we found that global mean FA and MT variation were only weakly related to TTV variation-with both measures of white matter microstructure showing negative scaling relationships with TTV [scaling coefficients were small in magnitude, but were significantly different from nulls of both 1 (linear scaling) and 0; Table 2; Fig. 4A]. Furthermore, in contrast to the regionally heterogeneous nature of WMV scaling, both FA-TTV and MT-TTV scaling relationships were profoundly negative throughout all white matter regions (i.e., significantly $>0$, but significantly $<1$; Figs. $4 B, 5$ ). Quantitative analysis further reinforced that the spatial patternings of voxel-wise scaling coefficients for local WMV, local FA, and local MT scaling were not highly correlated (or, at least, not as highly correlated as WMV scaling was across datasets; $r_{W M V-F A}=0.02, p>0.05 ; r_{M T-F A}$ $=0.34, p<0.01 ; r_{M T-W M V}=0.35, p<2.2 \times 10^{-16}$; Fig. $4 C$ ).

Finally, supplemental analyses in the NSPN dataset revealed that, for all three modalities examined, regional scaling results were highly consistent across images with different imposed voxel sizes (Warling, 2021a). This suggests our findings were not impacted by potential partial voluming effects. Supplementary analyses also established that cross voxel variation in scaling was highly correlated between analyses using TTV and GMV as the global metric $\left(r_{W M V}=0.70\right.$; Warling, 2021c). As expected given the small positive scaling between total WMV and TTV (Table 2), global and voxel-wise scaling coefficients for WMV were lower with total GMV than with TTV (global scaling of WMV with GMV =0.91; global scaling of WMV with TTV = 1.10; for regional analyses, see Warling, 2021c).

All regional WMV, FA, and MT maps, as well as conjunction maps, are publicly available on NeuroVault (https://identifiers. org/neurovault.collection:9115).

Local gray matter scaling correlates with local white matter scaling

Having localized regions of nonlinear WMV scaling, and also characterized the distinctness of these scaling patterns from those of white matter microstructure, we next examined how regional WMV scaling relationships might integrate with previouslyreported regional gray matter scaling relationships (Reardon et al., 2018). To achieve this, we considered the set of white matter voxels showing statistically-significant positive scaling with TTV in the HCP cohort (Fig. 1B, regions highlighted in red). Using a standard cortical parcellation (Desikan et al., 2006) in the IIT Human Brain Atlas (Zhang and Arfanakis, 2018), we identified the percent of all white matter "streamlines" entering or exiting a given gray matter region that passed through this set of positively-scaling white matter voxels. Then, we were able to test, and verify, that those cortical regions which scale more positively (i.e., have a greater proportion of positively scaling vertices) also tend to show a greater percent connectivity through positivelyscaling white matter voxels (Fig. 6A; Movie 1, red mask). For example, the inferior temporal region of the right hemisphere shows predominantly positive areal scaling with brain size, and is also highly connected through regions of positive WMV scaling. This finding suggests a coordinated pattern of anatomic scaling in the human brain, where gray matter regions that become proportionally larger in larger brains tend to be connected by white matter tracts that also become proportionally larger in larger brains. Among those cortical regions with the most marked positive areal scaling (i.e., over $50 \%$ of vertices scaling positively; Fig. $6 B$ ), we found that the regions most highly interconnected by positively-scaling white matter tracts were the right caudal middle frontal gyrus, right caudal anterior cingulate cortex, and left isthmus cingulate cortex (Fig. 6C).

\section{Discussion}

By integrating data across distinct cohorts and brain phenotypes, our study provides several novel insights into regional white matter scaling of the human brain. We consider each below.

Across all three datasets considered, we find that total WMV scales positively with brain size, while total GMV scales negatively. Consistent with previous findings in healthy cohorts (Lüders et al., 2002; de Jong et al., 2017), these relationships were not significantly modified by age or sex, despite known sex-and age-based differences in absolute global cortical measures (Cosgrove et al., 2007; Ritchie et al., 2018; Kaczkurkin et al., 


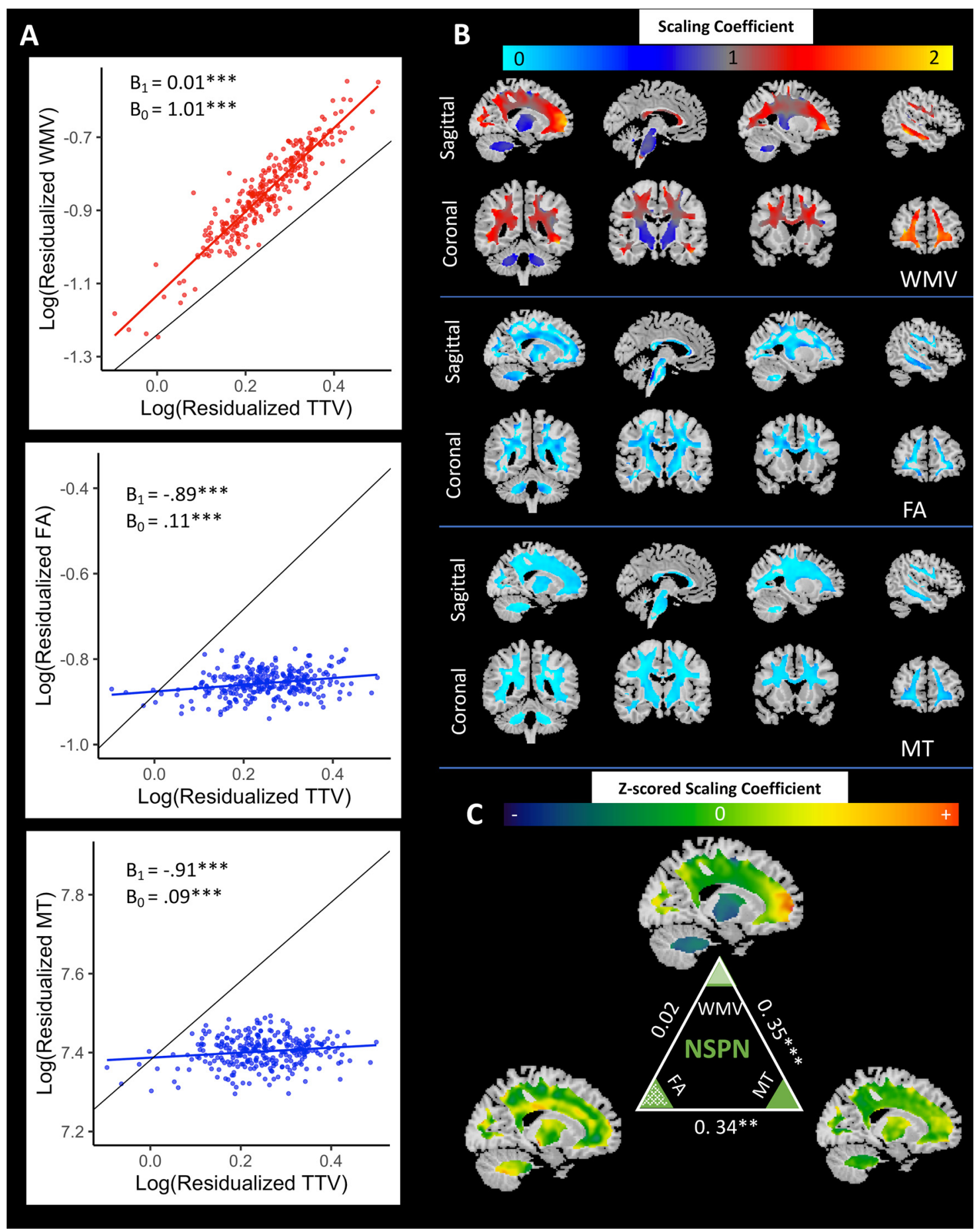

Figure 4. Nonlinear scaling of local fractional anisotropy (FA) and local magnetization transfer (MT) with brain size in the Neuroscience in Psychiatry Network (NSPN) cohort. A, Scatterplots showing scaling relationships between global white matter volume (WMV) and total tissue volume (TTV) (top), global mean FA and TTV (middle), and global MT and TTV (bottom) in the NSPN cohort (each dot is one participant). Positive and negative scaling relationships are denoted in red and blue (respectively), relative to the null of linear scaling (black line, slope $=1$ ); $\beta$ values and $p$ values are provided for comparisons between fit lines and null slopes of 1 (linear scaling) and 0 (no linear relationship between variables). B, Sagittal and coronal slices displaying the unthresholded map of local WMV, FA, and MT scaling. Although there are regions of both positive and negative WMV scaling, MT and FA scaling is uniformly negative. C, Correlations between voxel-wise MT, FA, and WMV scaling coefficients $\left(r_{\text {WMV-FA }}=0.02, p>0.05 ; r_{\text {MT-FA }}=0.34, p<0.01 ; r_{\text {MT-WMV }}=0.35, p<2.2 \times 10^{-16}\right)$, as well as Z-scored maps of these coefficients provided to aid comparison of relative regional scaling. ${ }^{*} p<0.05$, ${ }^{* *} p<0.01$, ${ }^{* * *} p<0.001$.

2019). Positive scaling relationships between total WMV and brain size have also been noted across mammalian species (Zhang and Sejnowski, 2000; Herculano-Houzel et al., 2010) and within the primate clade (Mota et al., 2019). Thus, our findings reinforce the notion that, in primates, possession of a larger brain size, through interspecies or interindividual variation, is accompanied by possession of proportionally larger WMV.

Moving beyond analysis of total WMV to voxel-level data, we show that the positive scaling of total WMV is underpinned by spatially heterogeneous, but highly replicable, regional WMV scaling patterns. This includes positive 


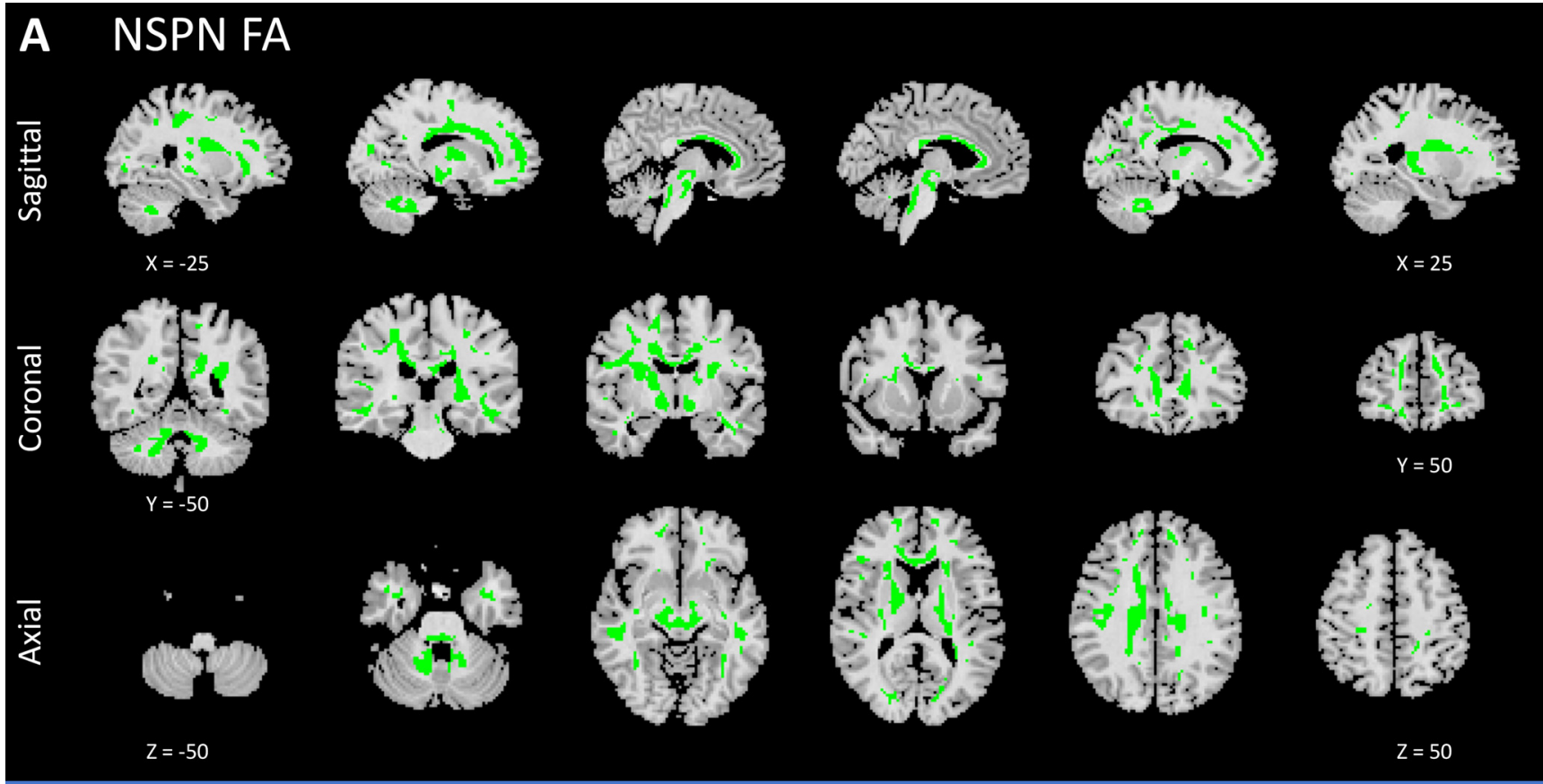

\section{B NSPN MT}
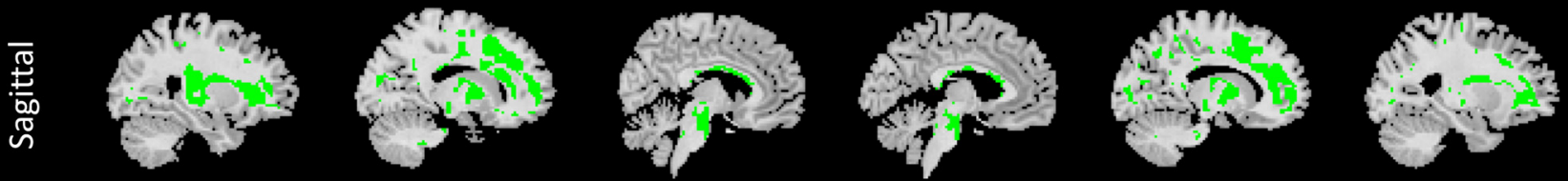

$X=-25$
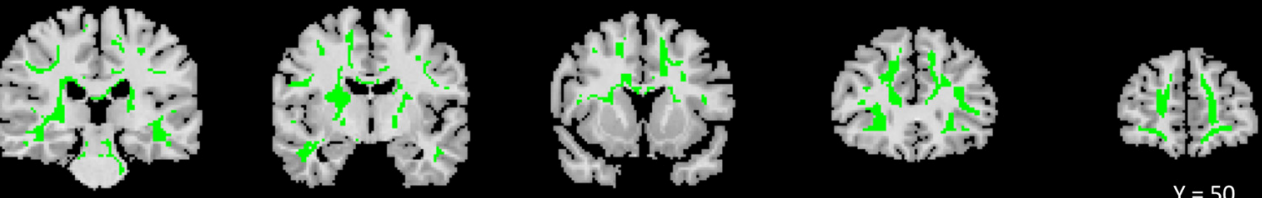

$\mathrm{Y}=50$
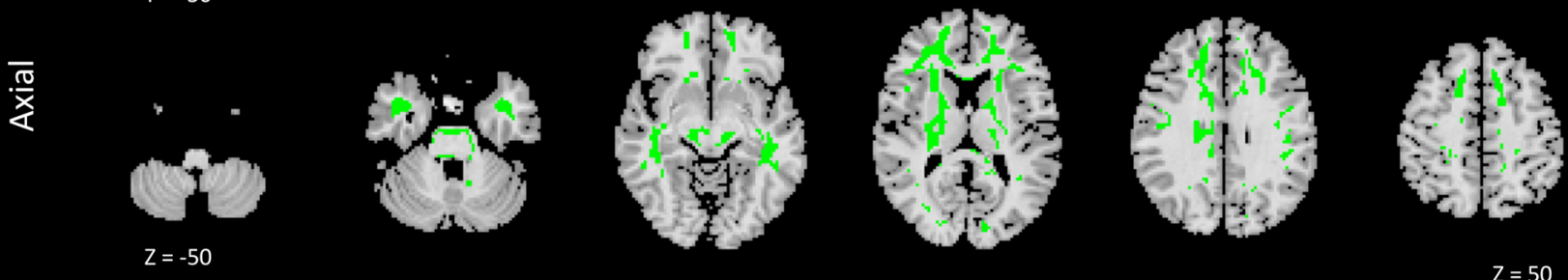

Figure 5. Thresholded maps showing significant linear associations of local fractional anisotropy (FA) and magnetization transfer (MT) with brain size in the Neuroscience in Psychiatry Network (NSPN) cohort. Sagittal, coronal, and axial slices displaying thresholded maps (FDR correction for multiple comparisons) of where regression coefficients (in log-log space) for FA ( $\boldsymbol{A}$ ) and MT $(\boldsymbol{B})$ with total tissue volume (TTV) are greater than zero.

WMV scaling in many cortical areas (i.e., subregions of the prefrontal, parietal, and occipital cortices, the anterior corona radiata, the superior longitudinal fasciculus, and the genu and splenium of the corpus callosum), alongside negative scaling in several subcortical regions (i.e., tracts coursing through the internal capsule, brainstem, and cerebellum). Notably, the striking consistency in regional scaling patterns across the three datasets was observed despite substantial differences in sample composition, scanning platforms, and image acquisition resolutions.

There are no other spatially comprehensive maps of WMV scaling with which to directly compare our findings. Current models of WMV scaling, based on early theories about hemispheric connectivity differences across brains of varying sizes (Ringo, 1991; Ringo et al., 1994), reports showing that corpus callosum volume scales negatively with TTV, both between primates (Rilling and Insel, 1999) and among humans (Jancke, 1997), diffusion imaging studies (Hänggi et al., 2014; Assaf et al., 2020), and functional connectivity work (Karolis et al., 2019), posit that larger brains should exhibit relatively decreased interhemispheric connectivity and increased intrahemispheric connectivity. However, assuming that relative expansion of WMV can be interpreted as promoting connectivity, our findings challenge this model on two grounds: we observe localized 

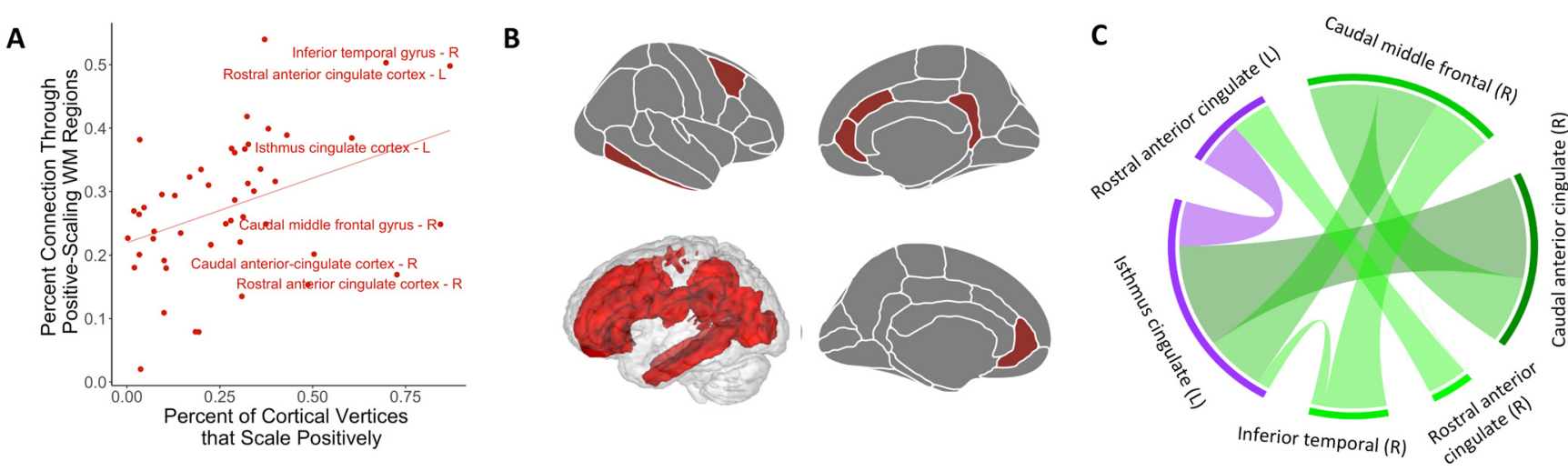

Figure 6. Coordination between gray and white matter scaling in the human brain. A, For each cortical region of interest (ROI) in the Desikan-Killiany Atlas, we used The IIT Human Brain Atlas to estimate the percent of white matter streamlines coming to or from that Rol that pass through the regions of significant positive white matter volume (WMV) scaling in the Human Connectome Project (HCP) (shown in Fig. 1B). As the scatterplot shows, this value was significantly correlated with the percent of all cortical vertices in that ROl that scaled positively in the HCP cohort in Reardon et al. (2018; $r=0.248, p=0.04)$, suggesting that allometric scaling of white matter in the human brain occurs in concert with the scaling pattern of associated gray matter regions. The names of the six most positively-scaling gray matter regions (i.e., those with $>50 \%$ positively scaling vertices) are highlighted. $\boldsymbol{B}$, Cortical surface (top right and left, bottom right) and tract (bottom left) maps showing, respectively, the locations of the same six most positively-scaling gray matter regions, and a mask of all positively-scaling WMV voxels in this cohort. C, Chord diagram showing strength of white matter connections through the WMV mask between the same six most positively-scaling gray matter regions. Edge size is proportional to number of white matter connections, and color denotes hemisphere identity (purple $=$ left; green $=$ right).

positive, rather than negative, WMV scaling within the corpus callosum, and we observe both positive and negative WMV scaling in intrahemispheric tracts, not homogenous negative WMV scaling.

Although our findings do not cohere with the notion that brain size-related white matter alterations are driven by the distinction between interhemispheric versus intrahemispheric tracts, they do suggest an alternative patterning principle: the distinction between higher-order associative versus lower-order sensorimotor systems. This principle is supported by several lines of empirical evidence. First, almost all regions of positive WMV scaling were within tracts mediating long-range frontal, parietal and temporal cortical connectivity [i.e., genu and splenium of the corpus callosum (Friedrich et al., 2020), superior longitudinal fasciculus (Kamali et al., 2014), cingulum (Bubb et al., 2018)]. Additionally, cortical regions with more pronounced positive gray matter scaling (Reardon et al., 2018) tend to connect more highly through positively-scaling white matter tracts (Fig. 6). Second, all regions of negative WMV scaling were localized to tracts mediating connectivity between lower-order sensory and motor systems [i.e., internal capsule (Kim et al., 2008), dorsalcolumn medial lemniscus (Purves et al., 2001), white matter underlying primary sensory cortex], which also themselves show negative anatomic scaling (Reardon et al., 2018). Thus, our findings propose a model of coordinated gray and white matter scaling, wherein (1) a distributed network of higher-order associative cortices and the white matter tracts connecting them become relatively expanded; while (2) another set of regions and tracts within motor and sensory systems becomes relatively diminished. The cortical aspects of this scaling program have already been demonstrated across all three major axes of primate brain size variation [evolutionary expansion (Hill et al., 2010), developmental growth (Hill et al., 2010), and interindividual variation (Reardon et al., 2018)]. Thus, these regional WMV scaling patterns may also be apparent in evolutionary (i.e., interspecies) and developmental contexts. There are hints that this prediction may hold, given evidence of disproportionate frontal lobe expansion (Schoenemann et al., 2005; Smaers et al., 2010; Barrett et al., 2020), and increased centrality of multimodal area interconnectivity (Ardesch et al., 2019), in humans compared with other primates. Integrating evolutionary and developmental aspects of local white matter scaling is an imporant goal for future research. These efforts should distinguish between questions about (1) the spatial similarity of regional developmental, interspecific, and intraspecific white matter scaling; and (2) the degree to which these scaling patterns share common mechanistic bases. Future white matter scaling work should also more closely examine its coordination with gray matter scaling, ideally at the voxel-level, by incorporating newer measures of white matter microstructure such as neurite orientation dispersion and density imaging (NODDI; Zhang et al., 2012).

Our findings also provide insight into potential developmental and histologic causes of regional WMV scaling. First, we verify that interindividual volume variation within regions of positive and negative WMV scaling is highly heritable. This is not necessarily surprising, as global brain volume variation is highly heritable (Peper et al., 2007), but does suggest that the stereotyped nature of regional WMV scaling may reflect genetic control. Second, we demonstrate nearly-universal negative FATTV and MT-TTV scaling relationships (e.g., hypolinear increases in FA and MT with increased brain size) at the global and regional levels. These findings are in line with prior reports which have also demonstrated absolute increases in FA and MT in larger brains (Takao et al., 2011, 2014), but stand in strong contrast to the positive (e.g., hyperlinear increases) in global WMV-TTV and heterogeneous regional WMV-TTV scaling relationships we observed. This inconsistency suggests that aspects of white matter organization thought to correlate with FA and MT (i.e., axonal integrity, fiber density, tract crossing, and myelination; Beaulieu, 2002; Lema et al., 2017) are unlikely to be immediate drivers of WMV scaling, and raises doubts regarding previously-hypothesized drivers of positive white matter scaling in primates [e.g., increased axonal branching (Zhang and Sejnowski, 2000), increased axonal diameter (Ringo et al., 1994; Changizi, 2001), increases in the proportion of myelinated axons (Wang et al., 2008), or increases in the degree of axonal myelination (i.e., decreased $g$ ratio; Paus, 2009)] because changes in those microstructural features would presumably dictate FA and MT scaling patterns that more closely replicate WMV scaling. Instead, we tentatively speculate that positive WMV scaling may reflect an increase in the number of axonal projections in a given white matter tract (Prothero, 1997). This speculation also 
aligns with the notion that regional differences in neuronal count may underlie the positive scaling of association cortices (Reardon et al., 2018) interconnected by these positively scaling white matter tracts. Testing this hypothesis, however, would require multimodal imaging studies and/or postmortem histologic analysis across differently-sized brains.

The "cause" of WMV scaling can also be considered from a teleological or functional perspective. Several observations in our study suggest that WMV scaling is functionally adaptive, including the reproducible nature of WMV scaling across different cohorts, the differential patterning of WMV scaling across associative versus sensorimotor systems, and the disproportionate expansion of total WMV in larger brains, despite the increased wiring costs this presumably occurs (Bullmore and Sporns, 2012). It is notable, therefore, that, similarly to what has been seen in gray matter (Reardon et al., 2018), WMV in positivelyscaling regions had only a weak, and negative, correlation with general cognitive ability after controlling for TTV. However, the very existence of nonlinear white matter scaling indicates that shifts in TTV and regional WMV proportions are linked, and suggests that local WMV changes themselves may be integral to the association between greater TTV and improved cognitive ability. Thus, we speculate that preferential expansion of association tracts in larger brains may contribute to the positive correlation between brain size and cognitive ability, but that possession of greater regional WMV volume than predicted by TTV may not provide additional functional advantages. Future work, however, would benefit from simultaneous measurement of multiple morphometric and cognitive features.

In summary, we systematically characterized regional white matter changes accompanying human brain size variation. We place our findings in the context of regional scaling for other brain phenotypes (FA and MT, and cortical surface area) and cognitive variation. Our analyses reveal that larger brains not only show a disproportionate expansion of associative cortices (Reardon et al., 2018) but also of the tracts that interconnect them. This knowledge helps refine current models of white matter scaling, indicates that brain size variation is linked to regional anatomic changes for both major brain tissue classes, and informs basic neuroscience by suggesting design principles required to support optimal brain functioning across natural brain size diversity. These basic science insights also provide a new context for interpreting regional WMV differences between groups that differ in brain size for demographic or clinical reasons.

\section{References}

Almasy L, Blangero J (1998) Multipoint quantitative-trait linkage analysis in general pedigrees. Am J Hum Genet 62:1198-1211.

Ardesch DJ, Scholtens LH, Li L, Preuss TM, Rilling JK, van den Heuvel MP (2019) Evolutionary expansion of connectivity between multimodal association areas in the human brain compared with chimpanzees. Proc Natl Acad Sci USA 116:7101-7106.

Ashburner J (2007) A fast diffeomorphic image registration algorithm. Neuroimage 38:95-113.

Ashburner J, Friston KJ (2005) Unified segmentation. Neuroimage 26:839885.

Assaf Y, Bouznach A, Zomet O, Marom A, Yovel Y (2020) Conservation of brain connectivity and wiring across the mammalian class. Nat Neurosci 23:805-808.

Barrett RLC, Dawson M, Dyrby TB, Krug K, Ptito M, D’Arceuil H, Croxson PL, Johnson PJ, Howells H, Forkel SJ, Dell'Acqua F, Catani M (2020) Differences in frontal network anatomy across primate species. J Neurosci 40:2094-2107.
Bates D, Eddelbuettel D (2013) Fast and elegant numerical linear algebra using the RccpEigen package. J Stat Soft 52:1024.

Beaulieu C (2002) The basis of anisotropic water diffusion in the nervous system - a technical review. NMR Biomed 15:435-455.

Blumenthal JD, Baker EH, Lee NR, Wade B, Clasen LS, Lenroot RK, Giedd JN (2013) Brain morphological abnormalities in 49,XXXXY syndrome: a pediatric magnetic resonance imaging study. Neuroimage Clin 2:197203.

Bubb EJ, Metzler-Baddeley C, Aggleton JP (2018) The cingulum bundle: anatomy, function, and dysfunction. Neurosci Biobehav Rev 92:104-127.

Bullmore E, Sporns O (2012) The economy of brain network organization. Nat Rev Neurosci 13:336-349.

Changizi MA (2001) Principles underlying mammalian neocortical scaling. Biol Cybern 84:207-215.

Cheverud JM (1982) Relationships among ontogenic, static, and evolutionary allometry. Am J Phys Anthropol 59:139-149.

Cosgrove KP, Mazure CM, Staley JK (2007) Evolving knowledge of sex differences in brain structure, function, and chemistry. Biol Psychiatry 62:847855.

Cox RW (1996) AFNI: software for analysis and visualization of functional magnetic resonance neuroimages. Comput Biomed Res 29:162-173.

de Jong LW, Vidal JS, Forsberg LE, Zijdenbos AP, Haight T, Sigurdsson S, Gudnason V, van Buchem MA, Launer LJ; Alzheimer's Disease Neuroimaging Initiative (2017) Allometric scaling of brain regions to intra-cranial volume: an epidemiological MRI study. Hum Brain Mapp 38:151-164.

Desikan RS, Ségonne F, Fischl B, Quinn BT, Dickerson BC, Blacker D, Buckner RL, Dale AM, Maguire RP, Hyman BT, Albert MS, Killiany RJ (2006) An automated labeling system for subdividing the human cerebral cortex on MRI scans into gyral based regions of interest. Neuroimage 31:968-980.

Dubois J, Galdi P, Paul LK, Adolphs R (2018) A distributed brain network predicts general intelligence from resting-state human neuroimaging data. Philos Trans R Soc Lond B Biol Sci 373:20170284.

Friedrich P, Forkel SJ, Thiebaut de Schotten M (2020) Mapping the principal gradient onto the corpus callosum. Neuroimage 223:117317.

Garcia de la Garza A, Vandekar S, Roalf D, Ruparel K, Gur R, Gur R, Satterthwaite T, Shinohara RT (2018) Voxel: mass-univariate voxelwise analysis of medical imaging data. $\mathrm{R}$ package version 1.3.5. Available at https://CRAN.R-project.org/package=voxel.

Giedd JN, Raznahan A, Alexander-Bloch A, Schmitt E, Gogtay N, Rapoport JL (2015) Child psychiatry branch of the National Institute of Mental Health longitudinal structural magnetic resonance imaging study of human brain development. Neuropsychopharmacology 40:43-49.

Glasser MF, Sotiropoulos SN, Wilson JA, Coalson TS, Fischl B, Andersson JL, Xu J, Jbabdi S, Webster M, Polimeni JR, Van Essen DC, Jenkinson M; WU-Minn HCP Consortium (2013) The minimal preprocessing pipelines for the Human Connectome Project. Neuroimage 80:105-124.

Gur RC, Butler ER, Moore TM, Rosen AFG, Ruparel K, Satterthwaite TD, Roalf DR, Gennatas ED, Bilker WB, Shinohara RT, Port A, Elliott MA, Verma R, Davatzikos C, Wolf DH, Detre JA, Gur RE (2021) Structural and functional brain parameters related to cognitive performance across development: replication and extension of the parieto-frontal integration theory in a single sample. Cereb Cortex 31:1444-1463.

Hagiwara A, Hori M, Kamagata K, Warntjes M, Matsuyoshi D, Nakazawa M, Ueda R, Andica C, Koshino S, Maekawa T, Irie R, Takamura T, Kumamaru KK, Abe O, Aoki S (2018) Myelin measurement: comparison between simultaneous tissue relaxometry, magnetization transfer saturation index, and T1w/T2w ratio methods. Sci Rep 8:10554.

Hänggi J, Fävenyi L, Liem F, Meyer M, Jancke L (2014) The hypothesis of neuronal interconnectivity as a function of brain size-a general organization principle of the human connectome. Front Human Neurosci 8:915.

Herculano-Houzel S, Mota B, Wong P, Kaas JH (2010) Connectivity-driven white matter scaling and folding in primate cerebral cortex. Proc Natl Acad Sci USA 107:19008-19013.

Hill J, Inder T, Neil J, Dierker D, Harwell J, Van Essen D (2010) Similar patterns of cortical expansion during human development and evolution. Proc Natl Acad Sci USA 107:13135-13140.

Huxley JS (1924) Constant differential growth-ratios and their significance. Nature 114:895-896.

Jancke L (1997) The relationship between corpus callosum size and forebrain. Cereb Cortex 7:48-56. 
Kaczkurkin AN, Raznahan A, Satterthwaite TD (2019) Sex differences in the developing brain: insights from multimodal neuroimaging. Neuropsychopharmacology 44:71-85.

Kamali A, Flanders AE, Brody J, Hunter JV, Hasan KM (2014) Tracing superior longitudinal fasciculus connectivity in the human brain using high resolution diffusion tensor tractography. Brain Struct Funct 219:269-281.

Karolis VR, Corbetta M, Thiebaut de Schotten M (2019) The architecture of functional lateralisation and its relationship to callosal connectivity in the human brain. Nat Commun 10:1417.

Kiddle B, Inkster B, Prabhu G, Moutoussis M, Whitaker KJ, Bullmore ET, Dolan RJ, Fonagy P, Goodyer IM, Jones PB (2018) Cohort profile: the NSPN 2400 cohort: a developmental sample supporting the Wellcome Trust NeuroScience in Psychiatry Network. Int J Epidemiol 47:18-19g.

Kim YH, Kim DS, Hong JH, Park CH, Hua N, Bickart KC, Byun WM, Jang SH (2008) Corticospinal tract location in internal capsule of human brain: diffusion tensor tractography and functional MRI study. Neuroreport 19:817-820.

Klein A, Andersson J, Ardekani BA, Ashburner J, Avants B, Chiang MC, Christensen GE, Louis Collins D, Gee J, Hellier P, Song JH, Jenkinson M, Lepage C, Rueckert D, Thompson P, Vercauteren T, Woods RP, John Mann J, Parsey RV (2009) Evaluation of 14 nonlinear deformation algorithms applied to human brain MRI registration. Neuroimage 46:786802.

Lema A, Bishop C, Malik O, Mattoscio M, Ali R, Nicholas R, Muraro PA, Matthews PM, Waldman AD, Newbould RD (2017) A comparison of magnetization transfer methods to assess brain and cervical cord microstructure in multiple sclerosis. J Neuroimaging 27:221-226.

Lenroot RK, Gogtay N, Greenstein DK, Wells EM, Wallace GL, Clasen LS, Blumenthal JD, Lerch J, Zijdenbos AP, Evans AC, Thompson PM, Giedd JN (2007) Sexual dimorphism of brain developmental trajectories during childhood and adolescence. Neuroimage 36:1065-1073.

Lüders E, Steinmetz H, Jäncke L (2002) Brain size and grey matter volume in the healthy human brain. Neuroreport 13:2371-2374.

Mädler B, Drabycz SA, Kolind SH, Whittall KP, MacKay AL (2008) Is diffusion anisotropy an accurate monitor of myelination? Correlation of multicomponent T2 relaxation and diffusion tensor anisotropy in human brain. Magn Reson Imaging 26:874-888.

Margulies DS, Ghosh SS, Goulas A, Falkiewicz M, Huntenburg JM, Langs G, Bezgin G, Eickhoff SB, Castellanos FX, Petrides M, Jefferies E, Smallwood J (2016) Situating the default-mode network along a principal gradient of macroscale cortical organization. Proc Natl Acad Sci USA 113:1257412579.

Mori S, Oishi K, Jiang H, Jiang L, Li X, Akhter K, Hua K, Faria AV, Mahmood A, Woods R, Toga AW, Bruce Pike G, Neto PR, Evans A, Zhang J, Huang H, Miller MI, van Zijl P, Mazziotta J (2008) Stereotaxic white matter atlas based on diffusion tensor imaging in an ICBM template. Neuroimage 40:570-582.

Mota B, Dos Santos SE, Ventura-Antunes L, Jardim-Messeder D, Neves K, Kazu RS, Noctor S, Lambert K, Bertelsen MF, Manger PR, Sherwood CC, Kaas JH, Herculano-Houzel S (2019) White matter volume and white/ gray matter ratio in mammalian species as a consequence of the universal scaling of cortical folding. Proc Natl Acad Sci USA 116:15253-15261.

Paus T (2009) Could sex differences in white matter be explained by g ratio? Front Neuroanat 3:14.

Peper JS, Brouwer RM, Boomsma DI, Kahn RS, Hulshoff Pol HE (2007) Genetic influences on human brain structure: a review of brain imaging studies in twins. Hum Brain Mapp 28:464-473.

Prothero J (1997) Scaling of cortical neuron density and white matter volume in mammals. J Hirnforsch 38:513-524.

Purves D, Augustine GJ, Fitzpatrick D, Katz LC, LaMantia A-S, McNamara JO, Mark Williams S (2001) The major afferent pathway for mechanosensory information: the dorsal column-medial lemniscus system. In: Neuroscience, Ed 2. Sunderland: Sinauer Associates.

R Core Team (2013) A language and environment for statistical computing. Vienna: R Foundation for Statistical Computing.

Reardon PK, Seidlitz J, Vandekar S, Liu S, Patel R, Park MTM, AlexanderBloch A, Clasen LS, Blumenthal JD, Lalonde FM, Giedd JN, Gur RC, Gur RE, Lerch JP, Chakravarty MM, Satterthwaite TD, Shinohara RT, Raznahan A (2018) Normative brain size variation and brain shape diversity in humans. Science 360:1222-1227.

Rilling JK, Insel TR (1999) Differential expansion of neural projection systems in primate brain evolution. Neuroreport 10:1453-1459.
Ringo JL (1991) Neuronal interconnection as a function of brain size. Brain Behav Evol 38:1-6.

Ringo JL, Doty RW, Demeter S, Simard PY (1994) Time is of the essence: a conjecture that hemispheric specialization arises from interhemispheric conduction delay. Cereb Cortex 4:331-343.

Ritchie SJ, Cox SR, Shen X, Lombardo MV, Reus LM, Alloza C, Harris MA, Alderson HL, Hunter S, Neilson E, Liewald DCM, Auyeung B, Whalley HC, Lawrie SM, Gale CR, Bastin ME, McIntosh AM, Deary IJ (2018) Sex differences in the adult huma brain: evidence from 5216 UK Biobank participants. Cereb Cortex 28:2959-2975.

Roalf DR, Quarmley M, Elliott MA, Satterthwaite TD, Vandekar SN, Ruparel K, Gennatas ED, Calkins ME, Moore TM, Hopson R, Prabhakaran K, Jackson CT, Verma R, Hakonarson H, Gur RC, Gur RE (2016) The impact of quality assurance assessment on diffusion tensor imaging outcomes in a large-scale population-based cohort. Neuroimage 125:903919.

Satterthwaite TD, Elliott MA, Ruparel K, Loughead J, Prabhakaran K, Calkins ME, Hopson R, Jackson C, Keefe J, Riley M, Mentch FD, Sleiman P, Verma R, Davatzikos C, Hakonarson H, Gur RC, Gur RE (2014) Neuroimaging of the Philadelphia Neurodevelopmental Cohort. Neuroimage 86:544-553.

Schmitt EJ, Raznahan A, Liu S, Neale MC (2020) The genetics of cortical myelination in young adults and its relationships to cerebral surface area, cortical thickness, and intelligence: a magnetic resonance imaging study of twins and families. Neuroimage 206:116319.

Schoenemann PT, Sheehan MJ, Glotzer LD (2005) Prefrontal white matter volume is disproportionately larger in humans than in other primates. Nat Neurosci 8:242-252.

Seidlitz J, Váša F, Shinn M, Romero-Garcia R, Whitaker KJ, Vértes PE, Wagstyl K, Kirkpatrick Reardon P, Clasen L, Liu S, Messinger A, Leopold DA, Fonagy P, Dolan RJ, Jones PB, Goodyer IM, Raznahan A, Bullmore ET; NSPN Consortium (2018) Morphometric similarity networks detect microscale cortical organization and predict inter-individual cognitive variation. Neuron 97:231-247.e7.

Smaers JB, Schleicher A, Zilles K, Vinicius L (2010) Frontal white matter volume is associated with brain enlargement and higher structural connectivity in anthropoid primates. PLoS One 5:e9123.

Sotiropoulos SN, Jbabdi S, Xu J, Andersson JL, Moeller S, Auerbach EJ, Glasser MF, Hernandez M, Sapiro G, Jenkinson M, Feinberg DA, Yacoub E, Lenglet C, Van Essen DC, Uğurbil K, Behrens TEJ; WUMinn HCP Consortium (2013) Advances in diffusion MRI acquisition and processing in the Human Connectome Project. Neuroimage 80:125-143.

Takao H, Hayashi N, Inano S, Ohtomo K (2011) Effect of head size on diffusion tensor imaging. Neuroimage 57:958-967.

Takao H, Hayashi N, Ohtomo K (2014) Sex dimorphism in the white matter: fractional anisotropy and brain size. J Magn Reson Imaging 39:917-923.

Tierney L, Rossini AJ, Li N, Sevcikova H (2018) Snow: simple network of workstations. $\mathrm{R}$ package version $0.4-3$. Available at https://CRAN.Rproject.org $/$ package $=$ snow.

Uğurbil K, Xu J, Auerbach EJ, Moeller S, Vu AT, Duarte-Carvajalino JM, Lenglet C, Wu X, Schmitter S, Van de Moortele PF, Strupp J, Sapiro G, De Martino F, Wang D, Harel N, Garwood M, Chen L, Feinberg DA, Smith SM, Miller KL, et al. (2013) Pushing spatial and temporal resolution for functional and diffusion MRI in the Human Connectome Project. Neuroimage 80:80-104.

Van Essen DC, Smith SM, Barch DM, Behrens TEJ, Yacoub E, Uğurbil K; WU-Minn HCP Consortium (2013) The WU-Minn Human Connectome Project: an overview. Neuroimage 80:62-79.

Ventura-Antunes L, Mota B, Herculano-Houzel S (2013) Different scaling of white matter volume, cortical connectivity, and gyrification across rodent and primate brains. Front Neuroanat 7:3.

Villalón-Reina JE, Martínez K, Qu X, Ching CRK, Nir TM, Kothapalli D, Corbin C, Sun D, Lin A, Forsyth JK, Kushan L, Vajdi A, Jalbrzikowski M, Hansen L, Jonas RK, van Amelsvoort T, Bakker G, Kates WR, Antshel KM, Fremont W, et al. (2020) Altered white matter microstructure in 22q11.2 deletion syndrome: a multisite diffusion tensor imaging study. Mol Psychiatry 25:2818-2831.

Walhovd KB, Fjell AM, Reinvang I, Lundervold A, Dale AM, Eilertsen DE, Quinn BT, Salat D, Makris N, Fischl B (2005) Effects of age on volumes of cortex, white matter and subcortical structures. Neurobiol Aging 26:1261-1270, discussion 1275-1278. 
Wang SSH, Shultz JR, Burish MJ, Harrison KH, Hof PR, Towns LC, Wagers MW, Wyatt KD (2008) Functional trade-offs in white matter axonal scaling. J Neurosci 28:4047-4056.

Warling A (2021a) Local scaling relationships across differing voxel sizes in the NSPN Cohort. figshare. Figure. Available at https://doi.org/10.6084/ m9.figshare.14364968.v2

Warling A (2021b) Heritability values for a variety of phenotypes in the HCP cohort. figshare. Figure. Available at https://doi.org/10.6084/ m9.figshare.14358530.v1

Warling A (2021c) Scaling relationships with total GMV in the NSPN cohort. Figshare. Figure. Available at https://doi.org/10.6084/m9.figshare.14758671.v1

Warnes GR, Bolker B, Lumley T (2020) gtools: various R programming tools. $\mathrm{R}$ package version 3.8.2. Available at https://cran.r-project.org/web/ packages/gtools/index.html.

Weiskopf N, Suckling J, Williams G, Correia MM, Inkster B, Tait R, Ooi C, Bullmore ET, Lutti A (2013) Quantitative multi-parameter mapping of $\mathrm{R} 1, \mathrm{PD}^{*}, \mathrm{MT}$, and R2* at 3T: a multi-center validation. Front Neurosci 7:95.
Whitcher B, Schmid VJ, Thornton A (2011) Working with the DICOM and NIfTI Data Standards in R. J Stat Soft 44:1-28.

Wickham H (2016) ggplot2: elegant graphics for data analysis. New York: Springer.

Wood SN (2011) Fast stable restricted maximum likelihood and marginal likelihood estimation of semiparametric and marginal likelihood estimation of semiparametric generalized linear models. J R Stat Soc B 73:3-36.

Zhang H, Schneider T, Wheeler-Kingshott CA, Alexander DC (2012) NODDI: practical in vivo neurite orientation dispersion and density imaging of the human brain. Neuroimage 61:1000-1016.

Zhang K, Sejnowski TJ (2000) A universal scaling law between gray matter and white matter of cerebral cortex. Proc Natl Acad Sci USA 97:56215626.

Zhang S, Arfanakis K (2018) Evaluation of standardized and study-specific diffusion tensor imaging templates of the adult human brain: template characteristics, spatial normalization accuracy, and detection of small inter-group FA differences. Neuroimage 172:40-50. 